\title{
FINITE ELEMENT METHODS ON NON-CONFORMING GRIDS BY PENALIZING THE MATCHING CONSTRAINT
}

\author{
ERIC BOILLAT ${ }^{1}$
}

\begin{abstract}
The present paper deals with a finite element approximation of partial differential equations when the domain is decomposed into sub-domains which are meshed independently. The method we obtain is never conforming because the continuity constraints on the boundary of the sub-domains are not imposed strongly but only penalized. We derive a selection rule for the penalty parameter which ensures a quasi-optimal convergence.
\end{abstract}

Mathematics Subject Classification. 65N12, 65N30, 65F10.

Received: June 25, 2002. Revised: December 17, 2002.

\section{INTRODUCTION}

In the standard finite element method (see e.g. [6]), the meshes have to satisfy the so-called Ciarlet's compatibility condition: if the intersection between two distinct elements is not empty, it has to be either a common vertex or a common edge or a common face. However, during the last ten years, many authors have proposed specially designed finite element methods allowing to use grids breaking the compatibility condition. These non-standard methods have many potential advantages. They can be applied with domains which are decomposed into regions meshed in a decoupled way. They can also be combined with economical adaptive procedures which do not lead to global contaminations of meshes.

The methods for solving second order p.d.e by the Galerkin technique on incompatible meshes can be classified into two categories: the conforming methods like the so-called "hanging node method" (see [12]) and the nonconforming methods. The latter will be the main topic of this article and their common feature can be described as follows. Consider a mesh consisting of several grids that are not compatible with each other and denote by $\Gamma$ the interfaces between those grids. The general idea of non-conforming methods is to avoid imposing the continuity of the test and trial functions across $\Gamma$ implicitly. It will only be imposed as a constraint. The consequence is that the corresponding variational formulation has the form of a saddle-point problem with a Lagrange multiplier in $H^{-\frac{1}{2}}(\Gamma)$. Its numerical resolution conceals the standard difficulty. It is necessary to find balanced pairs of discrete spaces for the Lagrange multiplier and for the original unknown. One of the most popular working pairs of discrete spaces is the mortar technique. A general presentation of the non-conforming mortar technique can be found in a series of articles (see $[3,10]$ or [2] and the references therein).

Keywords and phrases. Finite element methods, non-matching grids, penalty technique.

1 Department of Mechanical Engineering, Laboratory for Production Management and Process, EPFL, 1015 Lausanne,

Switzerland. e-mail: eric.boillat@epfl.ch 
In the present paper, we describe an alternative to the mortar technique for matching incompatible grids in the case of linear finite elements. The basic idea is to eliminate the Lagrange multiplier by means of the penalty technique. We get a discrete scheme which can be compared to the well-known Nitsche method (see [11]) without the consistency terms. The essential novelty of our work is to show that quasi-optimal convergence can be achieved with a penalty parameter proportional to the mesh size, and not to the square of the mesh size as a straightforward analysis tends to show. This result has two consequences. It improves the conditioning of the stiffness matrix. It also allows a numerical integration of the penalty term without any loss of convergence.

The plan of our work is as follows. In Section 2, we present our method on a simple model problem. In Section 3, the quasi-optimal convergence is proved. In Section 4, we simplify our method by integrating the penalty term numerically and we prove that the order of convergence is preserved. In Section 5, we explain how the stiffness matrix can be constructed and stored under the form of a sparse matrix. Finally, in Section 6 , we report some numerical experiments. They will demonstrate the efficiency of our method in a particular situation.

\section{Presentation of the Method}

In this section, our non-conforming method will be exposed on a model problem. Let us first introduce the needed notations.

\subsection{Notations}

For any open set $S \subset R^{d}, d=1,2,3$, and any $r \in \mathbb{R}$, we denote by $H^{r}(S)$ the usual Sobolev space: if $r \in \mathbb{N}$, $H^{r}(S)$ contains all the real-valued functions of $S$ with square-integrable derivatives up to order $r$, if $r \geq 0$ is non-integer, $H^{r}(S)$ is defined by interpolation and finally, if $r<0, H^{r}(S)$ is the dual space to the closure of $C_{0}^{\infty}(S)$ into $H^{r}(S)$. The symbol $\|\cdot\|_{r, S}$ denotes the usual norm in $H^{r}(S)$. The notation $L^{2}(S)$ is used instead of $H^{0}(S)$ and the scalar product in $L^{2}(S)$ will be referred to as $(\cdot, \cdot)_{S}$. For any $q \in \mathbb{N}, \mathbb{P}^{q}(S)$ is the set of the polynomial functions of degree $q$ over the domain $S$ and, for any $p, 1 \leq p<\infty, L^{p}(S)$ is the space of all the real-valued functions $u$ on $S$ such that $\|u\|_{L^{p}(S)}^{p} \equiv \int_{S}|u(x)|^{p} d x<\infty$. By means of parametrization, observe finally that the open curves in $\mathbb{R}^{2}$ are considered as open set in $\mathbb{R}^{1}$ and the open surfaces in $\mathbb{R}^{3}$ are considered as open set in $\mathbb{R}^{2}$.

\subsection{The functional framework}

Let $\Omega$ be a polygonal domain in $\mathbb{R}^{d}$ with $d=2$ or $d=3$ that can be divided into two non-overlapping polygonal and open sub-domains $\Omega_{\mathrm{c}}$ and $\Omega_{\mathrm{f}}$

$$
\bar{\Omega}=\overline{\Omega_{\mathrm{c}}} \cup \overline{\Omega_{\mathrm{f}}}, \quad \Omega_{\mathrm{c}} \cap \Omega_{\mathrm{f}}=\emptyset,
$$

the subdomain $\Omega_{\mathrm{c}}$ will be equipped with a coarse mesh while the subdomain $\Omega_{\mathrm{f}}$ will be equipped with a fine mesh. We finally denote by $\Gamma$ the open interface between the domains $\Omega_{\mathrm{c}}$ and $\Omega_{\mathrm{f}}$,

$$
\bar{\Gamma}=\overline{\Omega_{\mathrm{f}}} \cap \overline{\Omega_{\mathrm{c}}} .
$$

Remark 2.1. The reason why we have divided $\Omega$ into only two sub-domains is to keep the presentation as simple as possible. Our method can be applied even if $\Omega$ is divided into $M \geq 2$ non-overlapping polygonal sub-domains.

We now define the space $V$ as the set of all the piecewise $H^{1}$ functions over $\Omega$ :

$$
V=\left\{u: \Omega \rightarrow \mathbb{R} \mid u_{\mid \Omega_{\mathrm{c}}} \in H^{1}\left(\Omega_{\mathrm{c}}\right), u_{\mid \Omega_{\mathrm{f}}} \in H^{1}\left(\Omega_{\mathrm{f}}\right)\right\} .
$$


The space $V$ is an Hilbert space with the standard scalar product

$$
a(u, v)=(u, v)_{\Omega}+(\nabla u, \nabla v)_{\Omega}, u, v \in V
$$

and with the norm

The following estimate

$$
\|v\|_{V}^{2}=a(v, v)=\|v\|_{1, \Omega_{\mathrm{c}}}^{2}+\|v\|_{1, \Omega_{\mathrm{f}}}^{2}, v \in V .
$$

$$
\|v\|_{V} \leq\|v\|_{1, \Omega_{\mathrm{c}}}+\|v\|_{1, \Omega_{\mathrm{f}}} \leq \sqrt{2}\|v\|_{V}
$$

is a direct consequence of the definition (2.6) and of the Young's inequality. It will be used quite extensively. The jump operator across $\Gamma$ will be denoted by $B$. It maps continuously $V$ onto $H^{\frac{1}{2}}(\Gamma)$ and it is defined by

$$
B u(x)=u_{\mid \Omega_{\mathrm{f}}}(x)-u_{\mid \Omega_{\mathrm{c}}}(x), x \in \Gamma, u \in V .
$$

The kernel ker $B$ corresponds to the space $H^{1}(\Omega)$ (see [10]):

$$
H^{1}(\Omega)=\operatorname{ker} B .
$$

\subsection{The model problem}

Let $F \in L^{2}(\Omega)$ be given. We consider the functional

$$
J(u)=\frac{1}{2} a(u, u)-(F, u)_{\Omega}, u \in V .
$$

Our model problem is to minimize $J$ over $H^{1}(\Omega)$ :

$$
\text { find } u \in H^{1}(\Omega) \text { such that } J(u) \leq J(v), \forall v \in H^{1}(\Omega) \text {. }
$$

It is a standard variational formulation of an elliptic p.d.e with natural boundary conditions (see [6]):

$$
\begin{aligned}
u-\Delta u & =F, \text { in } \Omega, \\
\frac{\partial u}{\partial n} & =0, \text { on } \partial \Omega .
\end{aligned}
$$

Its solution exists and is unique. From now on, we will assume that it is regular:

$$
u \in H^{2}(\Omega)
$$

Remark 2.2. It may be observe that the regularity property (2.13) is always verified if the domain $\Omega$ is convex (see $[8])$.

Under the regularity assumption $(2.13)$, the equations $(2.11,2.12)$ are satisfied in the $L^{2}$-sense and the normal derivative of $u$ along the interface $\Gamma, \partial u / \partial n_{\mid \Gamma}$, is of class $H^{\frac{1}{2}}$ on each individual face of the polygonal boundary $\Gamma$. By a classical result about piecewise $H^{\frac{1}{2}}$ functions (see Th. 11.4 in Chap. 1 of [9]), we get that

$$
\frac{\partial u}{\partial n} \mid \Gamma_{\mid \Gamma} \in H^{\nu}(\Gamma)
$$

for any index $\nu$ between 0 and $\frac{1}{2}$, the value $\frac{1}{2}$ being excluded.

Because of the characterization $(2.8)$ of $H^{1}(\Omega)$, problem (2.10) may be seen as a constrained minimization problem. We want to find the minimum of $J$ in the space $V$ under the constraint

$$
B u=0 \text {. }
$$


To conclude this section, we use the regularity properties (2.13) and (2.14) to write the Euler equation for the solution $u$ to (2.10). With the convention that the unit normal $\mathbf{n}$ to $\Gamma$ is the outward normal to $\Omega_{\mathrm{f}}$, the Euler equation reads

$$
a(u, v)-\left(\frac{\partial u}{\partial n}{ }_{\mid \Gamma}, B v\right)_{\Gamma}=(F, v)_{\Omega}, v \in V .
$$

In (2.16), it can be observed that the normal derivative $\partial u / \partial n_{\mid \Gamma}$ is the Lagrange multiplier for the constraint (2.15).

\subsection{The numerical method}

We equip each sub-domains $\Omega_{\mathrm{c}}$ and $\Omega_{\mathrm{f}}$ with a regular family of meshes satisfying separately the Ciarlet's compatibility condition. They consist of triangles if $\overline{d=2}$ and of tetrahedrons if $d=3$. The precise definition of regularity and compatibility can be found in reference [6]. We use the notation $\mathcal{M}_{H}^{\mathrm{c}}$ to denote the meshes in $\Omega_{\mathrm{c}}$ and the notation $\mathcal{M}_{h}^{\mathrm{f}}$ for the meshes in $\Omega_{\mathrm{f}}$. The subscripts $H$ and $h$ represent the mesh sizes and are defined by

$$
H=\max _{K \in M_{h}^{c}} h(K) \text { and } h=\max _{K \in M_{h}^{\mathrm{f}}} h(K)
$$

where $h(K)$ denotes the size of any element $K$. It is of course assumed that

$$
H \geq h
$$

but the coarse mesh is nevertheless allowed to be locally finer than the fine mesh. On the other hand, denoting by $\rho(K)$ the roundedness of any element $K$, the regularity assumption means (see [6]) that there must be a constant $a>0$, independent of $H, h>0$, such that it holds

$$
\frac{\rho(K)}{h(K)} \geq a, \forall K \in \mathcal{M}_{H}^{\mathrm{c}} \cup \mathcal{M}_{h}^{\mathrm{f}} .
$$

The resulting mesh $\mathcal{M}_{\mathbf{h}}$,

$$
\mathcal{M}_{\mathbf{h}}=\mathcal{M}_{H}^{\mathrm{c}} \cup \mathcal{M}_{h}^{\mathrm{f}},
$$

where $\mathbf{h}$ stands for the pair $(H, h)$, is a grid which violates (in principle) the compatibility condition (see Fig. 1).

The trial space for the numerical method is the $\mathbb{P}^{1}$ finite element discretization of the space $V$,

$$
V_{\mathbf{h}}=\left\{u \in V \mid u_{\mid K} \in \mathbb{P}^{1}(K), \forall K \in \mathcal{M}_{\mathbf{h}}\right\}
$$

and the quadratic functional to minimize is

$$
J_{H}=J(u)+\frac{1}{2 H}\|B u\|_{0, \Gamma}^{2}, u \in V .
$$

The discrete problem for the approximation $u_{\mathbf{h}}$ to the solution $u$ of (2.10) thus reads

$$
\text { find } u_{\mathbf{h}} \in V_{\mathbf{h}} \text { such that } J_{H}\left(u_{\mathbf{h}}\right) \leq J_{H}(v), \forall v \in V_{\mathbf{h}} \text {. }
$$

Observe that $u_{\mathbf{h}}$ is solution to the variational equation

$$
a\left(u_{\mathbf{h}}, v\right)+\frac{1}{H}\left(B u_{\mathbf{h}}, B v\right)_{\Gamma}=(F, v)_{\Omega}, v \in V_{\mathbf{h}} .
$$

A straightforward analysis would lead to the conclusion that the factor in front of the penalty term $\|B u\|_{0, \Gamma}^{2}$ in (2.21) should be $\frac{1}{2 h^{2}}$ and not $\frac{1}{2 H}$ to ensure an optimal convergence of order $h$ (see Sect. II.4 in [5]). One of the major output of this paper will be to prove that the factor $\frac{1}{H}$ is sufficient, which is by far preferable since it limits the growth in the conditioning of the stiffness matrix. 


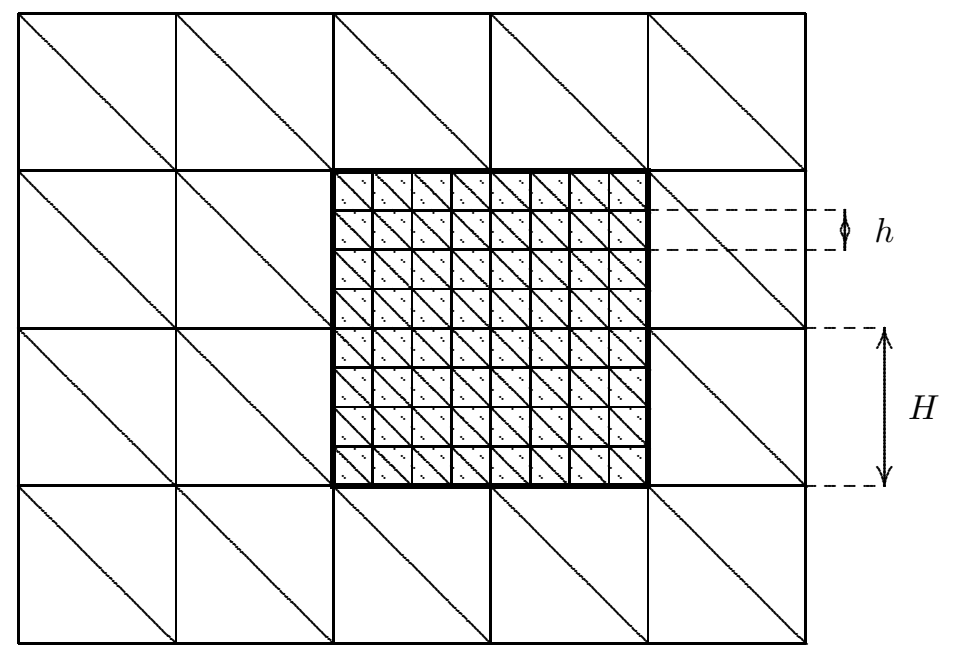

Figure 1. An example of a non-matching grid $\mathcal{M}_{\mathbf{h}}$ in two dimensions. The dashed region corresponds to the domain $\Omega_{\mathrm{f}}$.

Remark 2.3. Observe that the numerical method we are proposing is non-consistent. This can be seen by comparing the Euler equations (2.16) for $u$ and (2.23) for $u_{\mathbf{h}}$. To get a consistent and symmetric scheme, we should add a term like

$$
\left(\left\{\frac{\partial u_{\mathbf{h}}}{\partial n}\right\}, B v\right)_{\Gamma}+\left(\left\{\frac{\partial v}{\partial n}\right\}, B u_{\mathbf{h}}\right)_{\Gamma},
$$

on the left-hand side of $(2.23)\left(\{\cdot\}\right.$ denotes the average value on the interface: $\{g\}(x)=\frac{1}{2} g_{\mid \Omega_{\mathrm{c}}}(x)+\frac{1}{2} g_{\mid \Omega_{g}}(x)$, $x \in \Gamma)$. This operation leads to a well known method to approach boundary conditions and designed by Nitsche (see [11] or [13]). The error analysis of this method essentially amounts to the proof of stability and interpolation properties in well chosen norms. As we will see later on, the error analysis needed for the nonconsistent method (2.22) is more difficult.

However, the consistent Nitsche method has a severe drawback. The introduction of the term (2.24) in the stiffness matrix is a major overload. The computation of each stiffness element requires the integration over the interface $\Gamma$ of a combination of functions which are piecewise polynomials but on different meshes. Observe that this integration cannot be done numerically, because the term (2.24) is of highest degree.

In the next section, we study the convergence property of $u_{\mathbf{h}}$ to $u$ when the mesh sizes $h, H$ tend to zero.

\section{The COnvergence Result}

The error

$$
\left\|u-u_{\mathbf{h}}\right\|_{V}
$$

between the solution $u$ to (2.10) and the solution $u_{\mathbf{h}}$ to the discrete problem (2.22) will be estimated in two steps.

First step. We find a bound for the so called penalty error

$$
\left\|u-u_{H}\right\|_{V}
$$


where $u_{H}$ is the solution to the continuous and unconstrained problem which is to find

$$
u_{H} \in V \text { s.t. } J_{H}\left(u_{H}\right) \leq J_{H}(v), \forall v \in V \text {. }
$$

Let us point out that the notation $u_{H}$ does not refer to an approximation by means of the coarse grid. The subscript $H$ actually corresponds to the penalty term and it can be observed that $u_{H}$ satisfies the Euler equation

$$
a\left(u_{H}, v\right)+\frac{1}{H}\left(B u_{H}, B v\right)_{\Gamma}=(F, v)_{\Omega}, v \in V .
$$

Second step. We get a bound for the approximation error

$$
\left\|u_{H}-u_{\mathbf{h}}\right\|_{V}
$$

It will be obtained in a classical way, using that the unconstrained problem (3.3) is consistent with the discrete problem (2.22), as it can be observed by comparing the Euler equations (2.23) and (3.4).

In the sequel, the penalty norm, defined over the space $V$ by

$$
\|v\|_{H}^{2}=\|v\|_{V}^{2}+\frac{1}{H}\|B v\|_{0, \Gamma}^{2}, v \in V
$$

will be useful. Because of the obvious properties of the square root, observe that it holds

$$
\|v\|_{H} \leq\|v\|_{V}+\frac{1}{\sqrt{H}}\|B v\|_{0, \Gamma}, v \in V .
$$

\subsection{The penalty error}

In this paragraph, we analyse the penalty error (3.2). The result will be based on the regularity property (2.14) of the normal derivative $\partial u / \partial n_{\mid \Gamma}$.

Lemma 3.1. We assume that the solution $u$ to (2.10) verifies the regularity assumption (2.13). Then, for any index $\nu \in\left[0, \frac{1}{2}\right.$ ), there is a constant $C$ (depending only on $\nu$ and on the domains $\Omega_{\mathrm{c}}$ and $\Omega_{\mathrm{f}}$ ) such that the solution $u_{H}$ to (3.3) satisfies the estimate

$$
\left\|u_{H}-u\right\|_{V} \leq C H^{\frac{1}{2}+\nu}\left\|\frac{\partial u}{\partial n}\right\|_{\nu, \Gamma}, \forall H>0 .
$$

Proof. In the sequel, $\nu$ belongs to $\left[0, \frac{1}{2}\right)$ and $c$ is a generic constant. It can differ from line to line and may depend on $\nu$ and on the domains $\Omega_{\mathrm{c}}$ and $\Omega_{\mathrm{f}}$, but not on $H$.

Let us define a function $\mu$ on the boundary $\partial \Omega_{\mathrm{c}}$ of $\Omega_{\mathrm{c}}$. The function $\mu$ vanishes on $\partial \Omega_{\mathrm{c}} / \Gamma$ and corresponds to $\partial u / \partial n_{\mid \Gamma}$ on $\Gamma$. Since $\partial u / \partial n_{\mid \Gamma} \in H^{\nu}(\Gamma)$ with $\nu<\frac{1}{2}, \mu$ belongs to $H^{\nu}\left(\partial \Omega_{\mathrm{c}}\right)$ and satisfies the estimate $\|\mu\|_{0, \partial \Omega_{c}} \leq c\left\|\frac{\partial u}{\partial n \mid \Gamma}\right\|_{\nu, \Gamma}$ (see for instance Th. 11.4 in Chap. 1 of [9]). By means of a regularization procedure (based e.g. on truncated Fourier series), we can find a function $\lambda$ of class $H^{\frac{1}{2}}\left(\partial \Omega_{\mathrm{c}}\right)$ which is sufficiently close to $\mu$ in $L^{2}(\Gamma)$ :

$$
\begin{gathered}
\left\|\lambda-\frac{\partial u}{\partial n}\right\|_{0, \Gamma} \leq c H^{\nu}\left\|\frac{\partial u}{\partial n}\right\|_{\nu, \Gamma} \\
\|\lambda\|_{0, \partial \Omega_{c} / \Gamma} \leq c H^{\nu}\left\|\frac{\partial u}{\partial n}\right\|_{\nu, \Gamma}
\end{gathered}
$$


and with a norm in $H^{\frac{1}{2}}\left(\partial \Omega_{\mathrm{c}}\right)$ which can be controlled:

$$
\|\lambda\|_{\frac{1}{2}, \partial \Omega_{\mathrm{c}}} \leq c H^{\nu-\frac{1}{2}}\left\|\frac{\partial u}{\partial n} \mid \Gamma\right\|_{\nu, \Gamma} .
$$

Since the trace operator has a bounded right-inverse from $H^{\frac{1}{2}}\left(\partial \Omega_{\mathrm{c}}\right)$ into $H^{1}\left(\Omega_{\mathrm{c}}\right)$, there is a function $w \in H^{1}\left(\Omega_{\mathrm{c}}\right)$ which satisfies

and which is bounded by the norm of $\lambda$ :

$$
w_{\mid \partial \Omega_{\mathrm{c}}}=-\lambda
$$

$$
\|w\|_{1, \Omega_{\mathrm{c}}} \leq c\|\lambda\|_{\frac{1}{2}, \partial \Omega_{\mathrm{c}}} \leq c H^{\nu-\frac{1}{2}}\left\|\frac{\partial u}{\partial n}\right\|_{\nu, \Gamma} .
$$

The function $w: \Omega_{\mathrm{c}} \rightarrow \mathbb{R}$ may be extended by zero to the whole domain $\Omega$. We thus obtain a function $U$,

$$
U(x)= \begin{cases}w(x), & \text { if } x \in \Omega_{\mathrm{c}} \\ 0, & \text { if } x \in \Omega / \Omega_{\mathrm{c}},\end{cases}
$$

which belongs to $V$. Of course, $\|U\|_{V}=\|w\|_{1, \Omega_{c}}$ and it follows from (3.12) that

$$
\|U\|_{V} \leq c H^{\nu-\frac{1}{2}}\left\|\frac{\partial u}{\partial n}\right\|_{\nu, \Gamma}
$$

The value of the jump $B U$ (see definition (2.7)) of $U$ across the interface $\Gamma$ is easy to deduce from (3.13) and (3.11),

$B U=\lambda_{\mid \Gamma}$
and relation (3.8) gives an useful estimate for the $L^{2}$-norm of $B U-\partial u / \partial n_{\mid \Gamma}$ :

$$
\left\|B U-\frac{\partial u}{\partial n}\right\|_{\mid \Gamma}\left\|_{0, \Gamma} \leq c H^{\nu}\right\| \frac{\partial u}{\partial n} \|_{\nu, \Gamma}
$$

We now set

$$
z_{H}=u_{H}-u+H U
$$

and we prove the lemma in two steps. We first estimate $z_{H}$ in the space $V$. We then bound $u_{H}-u=z_{H}-H U$ by means of the triangle inequality.

First step. We start from the identity (see (3.6))

$$
\left\|z_{H}\right\|_{H}^{2}=a\left(z_{H}, z_{H}\right)+\frac{1}{H}\left(B z_{H}, B z_{H}\right)_{\Gamma} .
$$

In both terms $a\left(z_{H}, z_{H}\right)$ and $B\left(z_{H}, B z_{H}\right)_{\Gamma}$, we replace the first factor $z_{H}$ by its expression (3.17). We take the variational equation (3.4) for $u_{H}$ into account and we use that $B u=0$ because $u \in H^{1}(\Omega)$. Organizing the terms in a proper way, we get

$$
\left\|z_{H}\right\|_{H}^{2}=\left(F, z_{H}\right)_{\Omega}-a\left(u, z_{H}\right)+H a\left(U, z_{H}\right)+\left(B U, B z_{H}\right)_{\Gamma} .
$$

Because of the variational equation (2.16) for $u$, the two first terms on the right-hand side simplify to $-(\partial u / \partial n$, $\left.B z_{H}\right)_{\Gamma}$ and the expression for $\left\|z_{H}\right\|_{H}^{2}$ is:

$$
\left\|z_{H}\right\|_{H}^{2}=H a\left(U, z_{H}\right)+\left(B U-\partial u / \partial n, B z_{H}\right)_{\Gamma} .
$$


We use the Cauchy-Schwarz inequality to show that the right-hand side is less than

$$
H\|U\|_{V}\left\|z_{H}\right\|_{V}+\|B U-\partial u / \partial n\|_{0, \Gamma}\left\|B z_{H}\right\|_{0, \Gamma} .
$$

Applying Young's inequality and taking the definition (2.5) of $\left\|z_{H}\right\|_{H}^{2}$ into account, we get that

$$
\left\|z_{H}\right\|_{H}^{2} \leq c H^{2}\|U\|_{V}^{2}+c H\left\|B U-\frac{\partial u}{\partial n}\right\|_{0, \Gamma}^{2} .
$$

The estimates (3.14) for $U$ and (3.16) for $B U-\partial u / \partial n_{\mid \Gamma}$ now leads to the conclusion that

$$
\left\|z_{H}\right\|_{H} \leq c H^{\frac{1}{2}+\nu}\left\|\frac{\partial u}{\partial n}\right\|_{\nu, \Gamma} .
$$

Second step. Because of (3.17), we have $u_{H}-u=z_{H}-H U$. The triangle inequality yields

$$
\left\|u_{H}-u\right\|_{V} \leq\left\|z_{H}\right\|_{V}+H\|U\|_{V}
$$

and the lemma follows from this inequality and the estimates (3.18) for $\left\|z_{H}\right\|_{V} \leq\left\|z_{H}\right\|_{H}$ and (3.14) for $\|U\|_{V}$.

Remark 3.1. In Lemma 3.1, the order of convergence for $u_{H}$ to $u$ when the penalty parameter $H$ tend to zero is arbitrarily close to 1 . It is better than the classical convergence result of order $\frac{1}{2}$ which can be obtained in complete generality for the penalty technique (see Sect. II.4 in [5]).

The literature (see Prop. 4.1 of Sect. II.4 in [5] or Th. 3.1 in [4]) provides an order of convergence of exactly 1 . However, this result is only valid under some assumptions about the regularity of the Lagrange multipliers $\partial u / \partial n_{\Gamma}$, (see (2.16)) and about the constraint operator $B$ (e.g. the range of the adjoint operator of $B$ should be closed). In our framework, those assumptions are unfortunately not fulfilled.

\subsection{The approximation error}

Our error analysis is based on the observation that the discrete approximation $u_{\mathbf{h}}$ is the best approximation of $u_{H}$ with respect to the penalty norm (3.6). This remark is a direct consequence of the discrete Euler equation (2.23) and of the continuous Euler equation (3.4). Hence it holds

Lemma 3.2. $\left\|u_{H}-u_{\mathbf{h}}\right\|_{H} \leq \min _{v \in V_{\mathbf{h}}}\left\|u_{H}-v\right\|_{H}$.

Lemma 3.2 reduces the analysis of the approximation error to find a good interpolant in $V_{\mathbf{h}}$ for any $U \in V$ with respect to the penalty norm. This is done in the next lemma.

Lemma 3.3. For any $U \in V$, it holds

$$
\min _{v \in V_{\mathbf{h}}}\|U-v\|_{H} \leq C\left(\left\|U-U^{0}\right\|_{V}+H\left\|U^{0}\right\|_{2, \Omega_{\mathrm{c}}}+h\left\|U^{0}\right\|_{2, \Omega_{\mathrm{f}}}\right)
$$

where $U^{0}$ denotes any element of $H^{2}(\Omega)$ and where the constant $C<\infty$ only depends on the domains $\Omega_{\mathrm{c}}$ and $\Omega_{\mathrm{f}}$ and on the regularity assumptions (2.18).

Proof. In the sequel, $U$ is an element of $V, U^{0}$ is any element of $H^{2}(\Omega)$ and $c$ is a generic constant. The constant $c$ may differ from line to line but it only depends on the domains $\Omega_{\mathrm{c}}$ and $\Omega_{\mathrm{f}}$ and on the regularity assumption (2.18). In particular, it is independent of $h, H$ and of the pair $\left(U, U^{0}\right)$.

The idea of the proof is to find a good interpolant for $U^{0}$ in the penalty norm and to modify it slightly according to the jumps of $U$ across the interface $\Gamma$. 
First step. We define a function $v_{\mathbf{h}} \in V_{\mathbf{h}}$. In the domain $\Omega_{\mathbf{c}}, v_{\mathbf{h}}$ is the Lagrange interpolant to $U^{0}$ on the mesh $\mathcal{M}_{H}^{\mathrm{c}}$ and, in the domain $\Omega_{\mathrm{f}}, v_{\mathbf{h}}$ is the Lagrange interpolant to $U^{0}$ on the mesh $\mathcal{M}_{h}^{\mathrm{f}}$. The approximation properties of the Lagrange interpolant are easily deduced from standard scaling arguments (see [6]). They read

$$
\left\|U^{0}-v_{\mathbf{h}}\right\|_{1, \Omega_{\mathrm{c}}} \leq c H\left\|U^{0}\right\|_{2, \Omega_{\mathrm{c}}} \text { and }\left\|U^{0}-v_{\mathbf{h}}\right\|_{1, \Omega_{\mathrm{f}}} \leq c h\left\|U^{0}\right\|_{2, \Omega_{\mathrm{f}}}
$$

as well as

$$
\left\|U_{\mid \Omega_{\mathrm{c}}}^{0}-v_{\mathbf{h} \mid \Omega_{\mathrm{c}}}\right\|_{0, \Gamma} \leq c H^{\frac{3}{2}}\left\|U^{0}\right\|_{\frac{3}{2}, \Gamma} \text { and }\left\|U_{\mid \Omega_{\mathrm{f}}}^{0}-v_{\mathbf{h} \mid \Omega_{\mathrm{f}}}\right\|_{0, \Gamma} \leq c h^{\frac{3}{2}}\left\|U^{0}\right\|_{\frac{3}{2}, \Gamma} .
$$

We sum the two estimates in (3.20) and we use the inequality (2.6). We get that

$$
\left\|U^{0}-v_{\mathbf{h}}\right\|_{V} \leq c\left(H\left\|U^{0}\right\|_{2, \Omega_{\mathrm{c}}}+h\left\|U^{0}\right\|_{2, \Omega_{\mathrm{f}}}\right) \text {. }
$$

Because of the triangle inequality and of the embedding of $H^{2}\left(\Omega_{\mathrm{c}}\right)$ and of $H^{2}\left(\Omega_{\mathrm{f}}\right)$ into $H^{\frac{3}{2}}(\Gamma)$, we can deduce from (3.21) that

$$
\frac{1}{\sqrt{H}}\left\|B\left(U^{0}-v_{\mathbf{h}}\right)\right\|_{0, \Gamma} \leq c\left(H\left\|U^{0}\right\|_{2, \Omega_{\mathrm{c}}}+h \sqrt{\frac{h}{H}}\left\|U^{0}\right\|_{2, \Omega_{\mathrm{f}}}\right) .
$$

We add this result to (3.22) and we use that $H \geq h$ (see (2.17)). We conclude that

$$
\left\|U^{0}-v_{\mathbf{h}}\right\|_{V}+\frac{1}{\sqrt{H}}\left\|B\left(U^{0}-v_{\mathbf{h}}\right)\right\|_{0, \Gamma} \leq c\left(H\left\|U^{0}\right\|_{2, \Omega_{\mathrm{c}}}+h\left\|U^{0}\right\|_{2, \Omega_{\mathrm{f}}}\right)
$$

Second step. We now define another function $w_{\mathbf{h}} \in V_{\mathbf{h}}$. In the domain $\Omega_{\mathrm{c}}, w_{h}$ is the Clément's interpolant (see [7]) to $U-U^{0}$ on the mesh $\mathcal{M}_{H}^{\mathrm{c}}$ and, in the domain $\Omega_{\mathrm{f}}, w_{h}$ is the Clément's interpolant to $U-U^{0}$ on the mesh $\mathcal{M}_{h}^{\mathrm{f}}$. The standard property of the Clément's interpolant are summarized below:

$$
\begin{gathered}
\left\|w_{\mathbf{h}}\right\|_{1, \Omega_{\mathrm{c}}} \leq c\left\|U-U^{0}\right\|_{1, \Omega_{\mathrm{c}}} \text { and }\left\|w_{\mathbf{h}}\right\|_{1, \Omega_{\mathrm{f}}} \leq c\left\|U-U^{0}\right\|_{1, \Omega_{\mathrm{f}}} \\
\left\|U_{\mid \Omega_{\mathrm{c}}}-U_{\mid \Omega_{\mathrm{c}}}^{0}-w_{\mathbf{h} \mid \Omega_{\mathrm{c}}}\right\|_{0, \Gamma} \leq c H^{\frac{1}{2}}\left\|U-U^{0}\right\|_{1, \Omega_{\mathrm{c}}}
\end{gathered}
$$

and

$$
\left\|U_{\mid \Omega_{\mathrm{f}}}-U_{\mid \Omega_{\mathrm{f}}}^{0}-w_{\mathbf{h} \mid \Omega_{\mathrm{f}}}\right\|_{0, \Gamma} \leq \operatorname{ch}^{\frac{1}{2}}\left\|U-U^{0}\right\|_{1, \Omega_{\mathrm{f}}} .
$$

We sum the two relations in (3.24) and we use the estimate (2.6). We get

$$
\left\|w_{\mathbf{h}}\right\|_{V} \leq c\left\|U-U^{0}\right\|_{V}
$$

Since $U^{0}$ is of class $H^{1}$, the jump, $B U^{0}$, across the interface $\Gamma$ is zero. We thus can deduce from $(3.25,3.26)$ and from the triangle inequality that

$$
\frac{1}{\sqrt{H}}\left\|B\left(U-w_{\mathbf{h}}\right)\right\|_{0, \Gamma} \leq c\left(\left\|U-U^{0}\right\|_{1, \Omega_{\mathrm{c}}}+\sqrt{\frac{h}{H}}\left\|U-U^{0}\right\|_{1, \Omega_{\mathrm{f}}}\right) .
$$

We add this result to (3.27) and we use again that $H \geq h$ (see (2.17)). We finally obtain

$$
\left\|w_{\mathbf{h}}\right\|_{V}+\frac{1}{\sqrt{H}}\left\|B\left(U-w_{\mathbf{h}}\right)\right\|_{0, \Gamma} \leq c\left\|U-U^{0}\right\|_{V} .
$$

Conclusion. Let us set

$$
U_{h}=v_{\mathbf{h}}+w_{\mathbf{h}}
$$


Since $B U^{0}=0$, we can write

$$
\begin{aligned}
& \left\|U-U_{h}\right\|_{V}+\frac{1}{\sqrt{H}}\left\|B\left(U-U_{h}\right)\right\|_{0, \Gamma}= \\
& \quad\left\|\left(U-U^{0}\right)+\left(U^{0}-v_{\mathbf{h}}\right)-w_{\mathbf{h}}\right\|_{V}+\frac{1}{\sqrt{H}}\left\|B\left(U-w_{\mathbf{h}}\right)+B\left(U^{0}-v_{\mathbf{h}}\right)\right\|_{0, \Gamma} .
\end{aligned}
$$

By construction, $U_{h}$ is an element of $V$ and the left-hand side of (3.30) is a bound from above for $\min _{v \in V}\|U-v\|_{H}$ (see (3.7)). We can prove the lemma by applying the triangle inequality to the right-hand side of (3.30) and by combining the obtained result with the estimates (3.23) and (3.28).

From Lemmas 3.1-3.3 we can deduce the convergence property of our numerical method.

Theorem 3.1. We assume that the solution u to (2.10) verifies the regularity assumption (2.13). Then, for any index $\nu \in\left[0, \frac{1}{2}\right)$, there is a constant $C$, independent of the mesh sizes $h$ and $H$ and on the data $F$ to problem (2.10), such that the solution $u_{\mathbf{h}}$ to (2.23) satisfies the estimate

$$
\left\|u-u_{\mathbf{h}}\right\|_{V} \leq C\left(H^{\frac{1}{2}+\nu}\|u\|_{2, \Omega_{\mathrm{c}}}+h^{\frac{1}{2}+\nu}\|u\|_{2, \Omega_{\mathrm{f}}}\right) .
$$

Proof. Throughout the proof, $c$ is a generic constant which is independent of $h, H$ and $F$. It can be different from line to line and may depend on the domains $\Omega_{\mathrm{c}}$ and $\Omega_{\mathrm{f}}$ and on the regularity assumption (2.18).

Because of Lemma 3.2, the approximation error $\left\|u_{H}-u_{\mathbf{h}}\right\|_{H}$ may be bounded by the interpolation error for $u_{H}$ in the discrete space $V_{\mathbf{h}}$. We estimate the interpolation error by means of Lemma 3.3 with $U=u_{H}$ and $U^{0}=u$. We get that

$$
\left\|u_{H}-u_{\mathbf{h}}\right\|_{H} \leq c\left(\left\|u_{H}-u\right\|_{V}+H\|u\|_{2, \Omega_{\mathrm{c}}}+h\|u\|_{2, \Omega_{\mathrm{f}}}\right) .
$$

Because of the triangle inequality, $\left\|u-u_{\mathbf{h}}\right\|_{V} \leq\left\|u-u_{H}\right\|_{V}+\left\|u_{H}-u_{\mathbf{h}}\right\|_{V}$, and since the penalty norm is a bound for the $V$-norm (see (3.6)), we can deduce from (3.32) that

$$
\left\|u-u_{\mathbf{h}}\right\|_{V} \leq c\left(\left\|u_{H}-u\right\|_{V}+H\|u\|_{2, \Omega_{\mathrm{c}}}+h\|u\|_{2, \Omega_{\mathrm{f}}}\right) .
$$

Estimating $\left\|u-u_{H}\right\|_{V}$ by means of Lemma 3.1, we obtain that

$$
\left\|u-u_{\mathbf{h}}\right\|_{V} \leq c\left(H^{\frac{1}{2}+\nu}\left\|\frac{\partial u}{\partial n}\right\|_{\nu, \Gamma}+H\|u\|_{2, \Omega_{\mathrm{c}}}+h\|u\|_{2, \Omega_{\mathrm{f}}}\right) .
$$

for some given value of the index $\nu \in\left[0, \frac{1}{2}\right)$. The interface $\Gamma$ being a part of the boundary $\partial \Omega_{\mathrm{c}}$, it holds

$$
\left\|\frac{\partial u}{\partial n}\right\|_{\nu, \Gamma} \leq c\|u\|_{2, \Omega_{\mathrm{c}}}
$$

and we prove the Theorem by substituting this estimate into (3.33).

Remark 3.2. In the framework of the model problem $(2.11,2.12)$, it is known that the convergence order of the standard conforming FEM is 1 in the $H^{1}$-norm and 2 in the weaker $L^{2}$-norm (see [6]). Unfortunately, the convergence order of our non-conforming method (3.3) is not open to such an improvement. The reason is that $u_{\mathbf{h}}$ is actually an approximation of the solution $u_{H}$ to the penalty problem (3.4). If the approximation error $u_{\mathbf{h}}-u_{H}$ certainly converges at higher order in the $L^{2}$-norm, the situation is different for the penalty error $u_{H}-u$. As it can be seen from straightforward one-dimensional examples, the difference $u_{H}-u$ is in general of order $\leq 1$ with respect to $H$ in any kind of norms. 


\section{Numerical integration}

To simplify the stiffness matrix related to the discrete method (2.22), we propose a numerical integration for the penalty term $(B u, B v)_{\Gamma}$. We also prove that this operation does not destroy the convergence properties established in Theorem 3.1.

\subsection{The scheme with numerical integration}

We denote by $\Sigma_{H}$ the regular grid on the interface $\Gamma$ which is obtained by restricting the coarse mesh $\mathcal{M}_{H}^{\mathrm{f}}$ and we call $W_{H}$ the space of piecewise constant functions on the grid $\Sigma_{H}$ :

$$
W_{H}=\left\{v: \Omega \rightarrow \mathbb{R} \mid v_{\mid \gamma} \in \mathbb{P}^{0}(\gamma), \forall \gamma \in \Sigma_{H}\right\}
$$

The Lagrange interpolation operator onto the grid $\Sigma_{H}$ will be denoted by $r_{H}$. It is defined by

$$
\forall v \in C^{0}(\Gamma), r_{H} v \in W_{H} \text { and } r_{H} v\left(G_{\gamma}\right)=v\left(G_{\gamma}\right), \forall \gamma \in \Sigma_{H},
$$

where $G_{\gamma}$ is the center of gravity of the element $\gamma$. Finally, if $f$ maps $\Omega$ onto $\mathbb{R}$, the notation $\nabla_{\Gamma} f(x)$ will be used for the component of $\nabla f(x)$ collinear to $\Gamma$ at $x \in \Gamma$.

The discrete problem with numerical integration is to find $w_{\mathbf{h}} \in V_{\mathbf{h}}$ such that it holds

$$
a\left(w_{\mathbf{h}}, v\right)+\frac{1}{H} \int_{\Gamma} r_{H}\left(B w_{\mathbf{h}} B v\right) \mathrm{d} \sigma=(F, v)_{\Omega}, v \in V_{\mathbf{h}} .
$$

\subsection{Convergence analysis}

The convergence analysis for the method (4.3) will be based on two results. The first one is an error estimate for the numerical integration formula and can be proved by combining the Bramble lemma with a scaling argument.

Lemma 4.1. If $f: \Gamma \rightarrow \mathbb{R}$ is continuous and if $\nabla_{\Gamma} f$ is integrable, then it holds

$$
\int_{\Gamma}\left|f-r_{H} f\right| \mathrm{d} \sigma \leq C H \int_{\Gamma}\left|\nabla_{\Gamma} f\right| \mathrm{d} \sigma
$$

for some constant $c<\infty$ independent of $H$ and $f$.

The second result is a special case of inverse inequalities in spaces of piecewise polynomial functions. It is true in complete generality in the $2 d$-case, when the interface $\Gamma$ is a broken line. In a $3 d$-case, $\Gamma$ is a surface and we need a regularity hypothesis for the mesh $\Sigma_{H} \otimes \Sigma_{h}$ obtained by combining the two grids $\Sigma_{H}$ and $\Sigma_{h}$. The elements in $\Sigma_{H} \otimes \Sigma_{h}$ are obtained by intersecting the elements of $\Sigma_{H}$ with the elements of $\Sigma_{h}$. They are polygons. If $\tau \in \Sigma_{H} \otimes \Sigma_{h}$ we denote by $\alpha(\tau)$ its minimum angle and the assumption we need is a regularity hypothesis. There must be a constant $\alpha_{0}$, independent of $H, h>0$, such that it holds

$$
\alpha(\tau) \geq \alpha_{0}>0, \forall \tau \in \Sigma_{H} \otimes \Sigma_{h} .
$$

We now state the inverse inequality we are going to use.

Lemma 4.2. There is a constant $c<\infty$ depending only on the domain $\Omega$ and on the number $\alpha_{0}$ in hypothesis (4.5) such that it holds

for any $v, w \in V_{h}$.

$$
\int_{\Gamma}\left|\nabla_{\Gamma} B v B w\right| \mathrm{d} \sigma \leq c\|B v\|_{\frac{1}{2}, \Gamma}\|B w\|_{\frac{1}{2}, \Gamma},
$$


Proof. In that proof, $c$ denotes a constant depending only on $\Omega$ and $\alpha_{0}$ but which may differ from line to line. We only give the proof for the $3 d$-case. It can be easily transposed to the $2 d$-case.

Let $\tau \in \Sigma_{H} \otimes \Sigma_{h}$. Since $\nabla_{\Gamma} B v B w=B v \nabla_{\Gamma} B w+B v \nabla_{\Gamma} B w$, we can use the Hölder's inequality between $L^{4}$ and $L^{\frac{4}{3}}$ to get the estimate

$$
\int_{\tau}\left|\nabla_{\Gamma} B v B w\right| \mathrm{d} \sigma \leq\|B v\|_{L^{4}(\tau)}\left\|\nabla_{\Gamma} B w\right\|_{L^{\frac{4}{3}(\tau)}}+\|B w\|_{L^{4}(\tau)}\left\|\nabla_{\Gamma} B v\right\|_{L^{\frac{4}{3}(\tau)}} .
$$

The Sobolev imbedding of $H^{\frac{1}{2}}(\tau)$ into $L^{4}(\tau)$ (see Th. 7.57(a) in [1]) implies that

$$
\int_{\tau}\left|\nabla_{\Gamma} B v B w\right| \mathrm{d} \sigma \leq c\left(\|B v\|_{\frac{1}{2}, \tau}\left\|\nabla_{\Gamma} B w\right\|_{L^{\frac{4}{3}(\tau)}}+\|B w\|_{\frac{1}{2}, \tau}\left\|\nabla_{\Gamma} B v\right\|_{L^{\frac{4}{3}(\tau)}}\right) .
$$

Observe now that because of assumption (4.5) and of a standard scaling argument it holds

$$
\left\|\nabla_{\Gamma} f\right\|_{L^{\frac{4}{3}(\tau)}} \leq c\|f\|_{\frac{1}{2}, \tau}
$$

for any linear function $f$ defined over the polygon $\tau \in \Sigma_{H} \otimes \Sigma_{h}$. As $v$ and $w$ both belong to $V_{h}$, we may apply (4.8) to $f=B v$ and to $f=B w$ on the right-hand side of (4.7). The conclusion is that

$$
\int_{\tau}\left|\nabla_{\Gamma} B v B w\right| \mathrm{d} \sigma, \leq c\|B v\|_{\frac{1}{2}, \tau}\|B w\|_{\frac{1}{2}, \tau}, \forall \tau \in \Sigma_{H} \otimes \Sigma_{h}
$$

The lemma is now proved by summing the above relation for any $\tau \in \Sigma_{H} \otimes \Sigma_{h}$. We just have to use the Cauchy-Schwarz inequality to bound the sum $\sum_{\tau}\|B v\|_{\frac{1}{2}, \tau}\|B w\|_{\frac{1}{2}, \tau}$ appearing on the right-hand side by the product $\|B v\|_{\frac{1}{2}, \Gamma}\|B w\|_{\frac{1}{2}, \Gamma}$.

We now prove the convergence properties of the discrete scheme (4.3).

Theorem 4.1. We assume that the solution $u$ to (2.10) verifies the regularity assumption (2.13). Then, for any index $\nu \in\left[0, \frac{1}{2}\right)$, there is a constant $C$, independent of the mesh sizes $h$ and $H$ and of the data $F$ to problem (2.10), such that the solution $w_{\mathbf{h}}$ to (4.3) satisfies the estimate

$$
\left\|u-w_{\mathbf{h}}\right\|_{V} \leq C\left(H^{\frac{1}{2}+\nu}\|u\|_{2, \Omega_{\mathrm{c}}}+h^{\frac{1}{2}+\nu}\|u\|_{2, \Omega_{\mathrm{f}}}\right) .
$$

Proof. We choose the test function $v$ as $v=u_{\mathbf{h}}-w_{\mathbf{h}}$ in the Euler equations (2.23) and (4.3) for $u_{\mathbf{h}}$ and $w_{\mathbf{h}}$ and we subtract the result. We get that

$$
\left\|u_{\mathbf{h}}-w_{\mathbf{h}}\right\|_{V}^{2}-\frac{1}{H} \int_{\Gamma} r_{H}\left(B w_{\mathbf{h}} B\left(u_{\mathbf{h}}-w_{\mathbf{h}}\right)\right) \mathrm{d} \sigma+\frac{1}{H}\left(B u_{\mathbf{h}}, B\left(u_{\mathbf{h}}-w_{\mathbf{h}}\right)\right)_{\Gamma}=0 .
$$

Adding the term $\frac{1}{H} \int_{\Gamma} r_{H}\left(B u_{\mathbf{h}} B\left(u_{\mathbf{h}}-w_{\mathbf{h}}\right)\right) \mathrm{d} \sigma$ on both sides yields

$$
\left\|u_{\mathbf{h}}-w_{\mathbf{h}}\right\|_{V}^{2} \leq \frac{1}{H}\left(\int_{\Gamma} r_{H}\left(B u_{\mathbf{h}} B\left(u_{\mathbf{h}}-w_{\mathbf{h}}\right)\right) \mathrm{d} \sigma-\left(B u_{\mathbf{h}}, B\left(u_{\mathbf{h}}-w_{\mathbf{h}}\right)\right)_{\Gamma}\right) .
$$

Since $\left(B u_{\mathbf{h}}, B\left(u_{\mathbf{h}}-w_{\mathbf{h}}\right)\right)_{\Gamma}=\int_{\Gamma} B u_{\mathbf{h}} B\left(u_{\mathbf{h}}-w_{\mathbf{h}}\right) \mathrm{d} \sigma$, the right-hand side can be bounded by means of Lemmas 4.1 and 4.2 :

where $c$ does not depend on $H$ and $h$.

$$
\left\|u_{\mathbf{h}}-w_{\mathbf{h}}\right\|_{V}^{2} \leq c\left\|B u_{\mathbf{h}}\right\|_{\frac{1}{2}, \Gamma}\left\|B\left(u_{\mathbf{h}}-w_{\mathbf{h}}\right)\right\|_{\frac{1}{2}, \Gamma}
$$


Observe now that $B u_{\mathbf{h}}=B\left(u_{\mathbf{h}}-u\right)$, because $B u=0$ and recall that the jump operator $B$ is continuous from $V$ into $H^{\frac{1}{2}}(\Gamma)$. We thus can improve the estimate (4.10). It now reads $\left\|u_{\mathbf{h}}-w_{\mathbf{h}}\right\|_{V}^{2} \leq c\left\|u_{\mathbf{h}}-u\right\|_{V}\left\|u_{\mathbf{h}}-w_{\mathbf{h}}\right\|_{V}$ or, after dividing by $\left\|u_{\mathbf{h}}-w_{\mathbf{h}}\right\|_{V}$,

$$
\left\|u_{\mathbf{h}}-w_{\mathbf{h}}\right\|_{V} \leq c\left\|u_{\mathbf{h}}-u\right\|_{V} .
$$

In view of this relation, the Theorem 4.1 is a direct consequence of the triangle inequality and of the convergence properties of $u_{\mathbf{h}}$ proved in Theorem 3.1.

Remark 4.1. If we replace the factor $\frac{1}{H}$ in front of the penalty term in (4.3) by $\frac{a}{H}$, where $a$ is a positive constant independent of the mesh sizes $H$ and $h$, the error estimate (4.9) will still be valid. However, the constant $C$ involved in (4.9) will depend on $a$. An open question is to find the optimal value for $a$ such that $C=C(a)$ is minimum.

\section{IMPlementation OF THE PENALTy TERM IN THE STIFFNESS MATRIX}

In this section, we consider the part of the stiffness matrix which is related to the penalty term in (4.3). We explain how to construct it when the connectivity tables of the grid $\Sigma_{H}$ and of the grid $\Sigma_{h}$, obtained by restricting the meshes $\mathcal{M}_{H}^{\mathrm{c}}$ and $\mathcal{M}_{h}^{\mathrm{f}}$, are known. In a $3 d$-problem, we shall see that this operation amounts to localize a set of points in a triangular mesh.

If meas $(\gamma)$ and $C_{\gamma}$ denote the area and the center of any element $\gamma \in \Sigma_{H}$, the definition (4.2) of the interpolation operator $r_{H}$ reduces the penalty term to

$$
\int_{\Gamma} r_{H}(B w B v) \mathrm{d} \sigma=\sum_{\gamma \in \Sigma_{H}} \operatorname{meas}(\gamma) B w\left(C_{\gamma}\right) B v\left(C_{\gamma}\right), \forall w, v \in V_{\mathbf{h}}
$$

Let now $P_{1} \ldots P_{N}$ be the nodes in the non-compatible mesh $\mathcal{M}_{H}^{\mathrm{c}} \cup \mathcal{M}_{h}^{\mathrm{c}}$ and let $\varphi_{j}$ be the shape function associated to $P_{j}, j=1 \ldots N$ :

$$
\varphi_{j} \in V_{\mathbf{h}} \text { and } \varphi_{j}\left(P_{i}\right)=\delta_{i j}, i=1 \ldots N \text {. }
$$

If $\mathbf{w}$ and $\mathbf{v} \in \mathbb{R}^{N}$ are the components of the functions $w, v \in V_{\mathbf{h}}$ in the family of shape functions:

$$
w=\sum_{j=1}^{N} w_{j} \varphi_{j} \text { and } v=\sum_{j=1}^{N} v_{j} \varphi_{j}
$$

we can rewrite the relation (5.1) in a matrix form. It reads

$$
\int_{\Gamma} r_{H}(B w B v) \mathrm{d} \sigma=\mathbf{w}^{T}\left(\sum_{\gamma \in \Sigma_{H}} \operatorname{meas}(\gamma) \mathbf{b}(\gamma) \mathbf{b}(\gamma)^{T}\right) \mathbf{v} .
$$

In (5.3), the vector $\mathbf{b}(\gamma), \gamma \in \Sigma_{H}$, is defined by:

$$
b(\gamma)_{j}=B \varphi_{j}\left(C_{\gamma}\right)=\varphi_{j \mid \Omega_{\mathrm{f}}}\left(C_{\gamma}\right)-\varphi_{j \mid \Omega_{\mathrm{c}}}\left(C_{\gamma}\right), j=1 \ldots N
$$

and the part of the stiffness matrix related to the penalty term is exactly

$$
R=\sum_{\gamma \in \Sigma_{H}} \operatorname{meas}(\gamma) \mathbf{b}(\gamma) \mathbf{b}(\gamma)^{T}
$$

If we intend to solve the discrete problem (4.3) by means of an iterative scheme (like the conjugate gradient method), it is not necessary to construct $R$ explicitly. Storing the vectors $\mathbf{b}(\gamma)$ is enough and we benefit from 

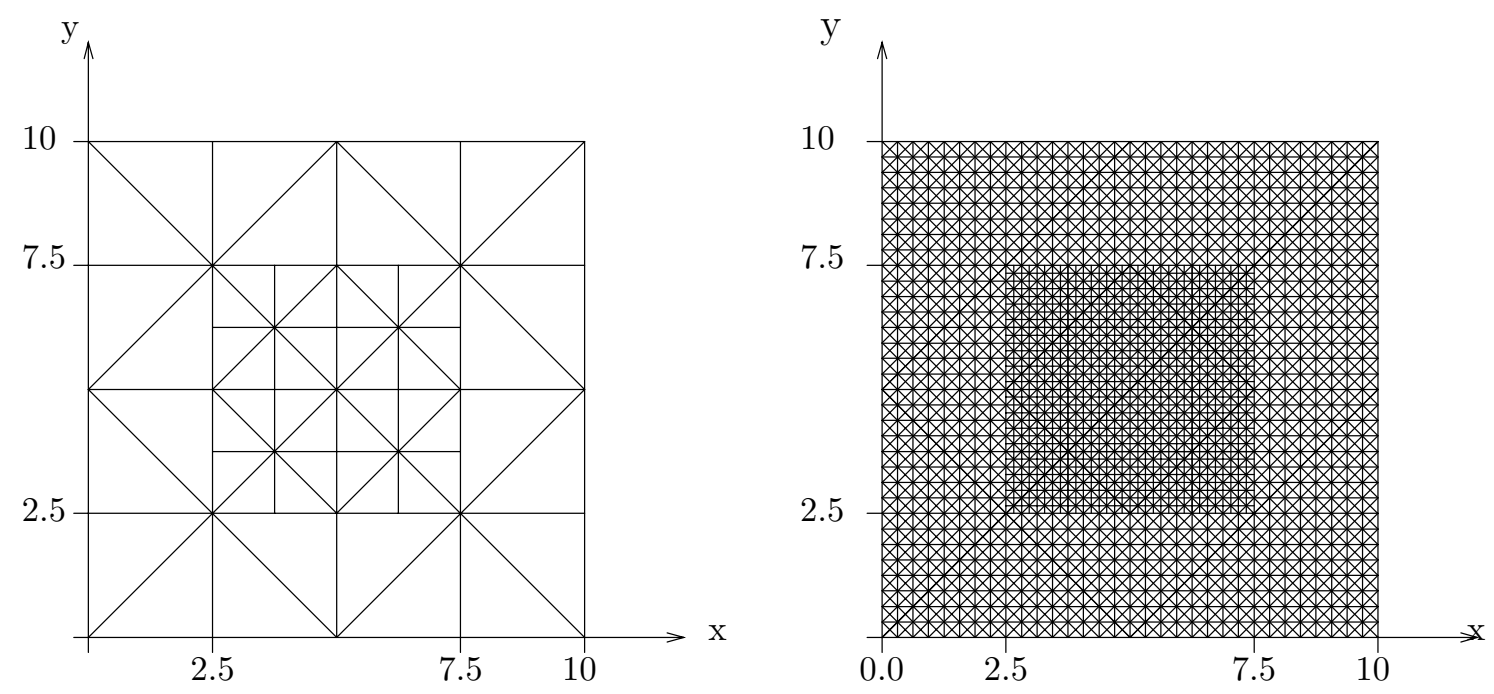

Figure 2. Examples of meshes used with the mortaring technique.

their sparse structure. For any $\gamma \in \Sigma_{H}$, the vector $\mathbf{b}(\gamma)$ has actually at most six non-zero components in a $3 d$-problem and only four in a $2 d$-problem.

If $\gamma$ is a given element of $\Sigma_{H}$ and if the problem is three-dimensional, the three first non-zero components of $\mathbf{b}(\gamma)$ correspond to the vertices $P_{K_{1}}, P_{K_{2}}$ and $P_{K_{3}}$ of the triangle $\gamma$. The indices $K_{1}, K_{2}$ and $K_{3}$ are of course available in the connectivity table and we have

$$
b(\gamma)_{K_{1}}=b(\gamma)_{K_{2}}=b(\gamma)_{K_{3}}=-\frac{1}{3} .
$$

To construct the three other non-zero components of $\mathbf{b}(\gamma)$, we have to find a triangle $\gamma^{\prime} \in \Sigma_{h}$ containing $C_{\gamma}$. Such a triangle exists and we denote its vertices by $P_{k_{1}}, P_{k_{2}}$ and $P_{k_{3}}$. Determining the indices $k_{1}, k_{2}$ and $k_{3}$ as well as the two coefficients $\xi, \eta \in[0,1]$ such that it holds

$$
O C_{\gamma}=\xi O P_{k_{1}}+\eta O P_{k_{1}}+(1-\xi-\eta) O P_{k_{3}}
$$

amounts to the classical problem of locating a point in a triangular mesh. Once $k_{1}, k_{2}, k_{3}, \eta$ and $\xi$ are known, we can set

$$
b(\gamma)_{k_{1}}=\xi, \quad b(\gamma)_{k_{2}}=\eta \quad \text { and } \quad b(\gamma)_{k_{3}}=1-\xi-\eta .
$$

\section{Numerical RESUlts}

The goal of this section is to demonstrate the efficiency of the numerical scheme (4.3). We consider the model problem $(2.11,2.12)$ in $d=2$ dimensions. The domain $\Omega$ is a square domain, $\Omega=(0,10)^{2}$, and the right-hand side $F$ is adjusted in such a way that the exact solution is the product of a polynomial factor (to fulfil the boundary condition (2.12)) and of a Gaussian factor of radius 1.25:

$$
u(x, y)=x^{2}(x-10)^{2} y^{2}(y-10)^{2} \exp -\frac{(x-5)^{2}+(y-5)^{2}}{(1.25)^{2}},(x, y) \in \Omega .
$$

The domain $\Omega_{\mathrm{f}}$, to be equipped with a fine mesh, is defined as the central square $\Omega_{\mathrm{f}}=(2.5,7.5)^{2}$ and the domain $\Omega_{\mathrm{c}}$, to be meshed coarsely, is the complementary of $\Omega_{\mathrm{f}}$ in $\Omega$ (see Fig. 2). 
TABLE 1. The value of the discretisation parameters.

\begin{tabular}{|l||l|l|l|l|l|l|l|}
\hline mesh & No. 1 & No. 2 & No. 3 & No. 4 & No. 5 & No. 6 & No. 7 \\
\hline \hline$H$ & 2.5000 & 1.7677 & 1.2500 & 0.8839 & 0.6250 & 0.4419 & 0.3125 \\
\hline$h$ & 1.2500 & 0.8839 & 0.6250 & 0.4419 & 0.3125 & 0.2210 & 0.1562 \\
\hline
\end{tabular}

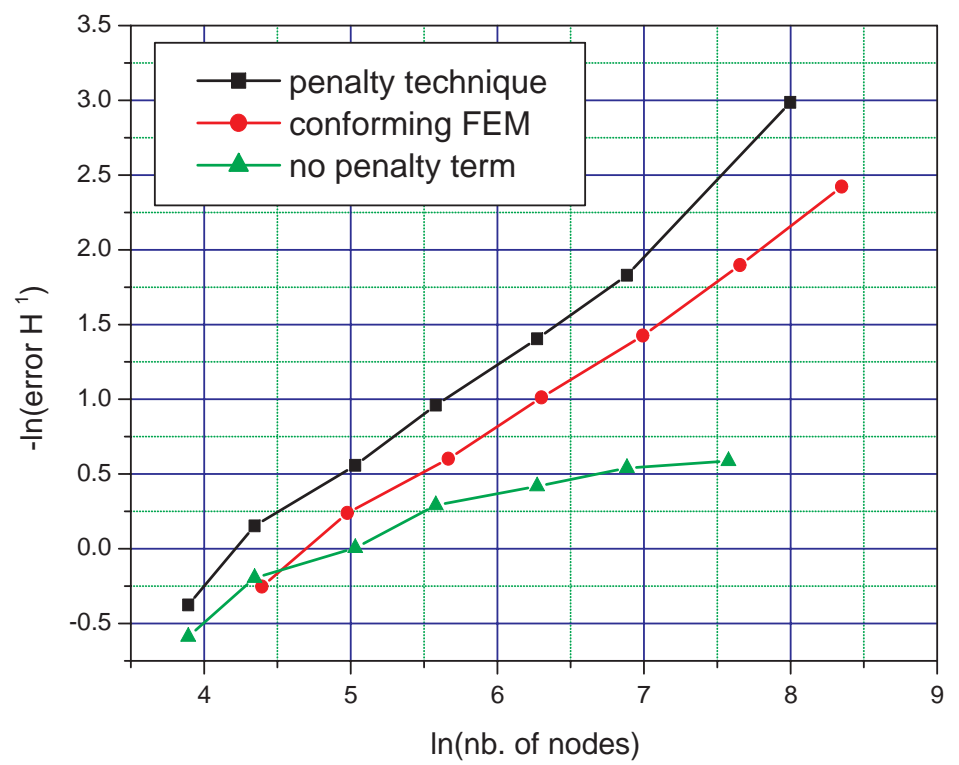

FiguRE 3. Error comparisons.

To approach the solution $u$ to the model problem $(2.11,2.12)$ with the non-conforming FEM (4.3), we meshed the two domains $\Omega_{\mathrm{f}}$ and $\Omega_{\mathrm{c}}$ independently. A rough analysis, based on the explicit formula (6.1), shows that the $H^{2}\left(\Omega_{\mathrm{f}}\right)$ semi-norm of $u$ is, at least, twice larger than its $H^{2}\left(\Omega_{\mathrm{c}}\right)$ semi-norm. To balance the two contributions in the error estimate (4.9), we thus kept a ratio of $1: 2$ between the fine mesh size, $h$, and the coarse mesh size, $H$. We computed the discrete solution to problem (4.3) for seven different meshes. The values of $H$ and $h$ which have been used can be found in Table 1 and the first and last meshes are represented in Figure 2. Observe also that the factor $\frac{1}{H}$ in front of the penalty term in (4.3) has been replaced by $\frac{10^{4}}{H}$ to improve convergence (see Rem. 4.1).

The error $\left\|u-w_{\mathbf{h}}\right\|_{1}$ between the exact solution $u$ to the model problem and its numerical approximation $\mathbf{w}_{h}$ is plotted in Figure 3 as a function of the number of nodes (log-log scale).

At the same time, we applied the standard conforming FEM to the problem $(2.11,2.12)$. We performed again seven different computations with seven different meshes. For the purpose of comparison, the $H^{1}(\Omega)$-norm of the error has been represented as a function of the number of nodes (log-log scale) in Figure 3 . The conclusion which we can draw is obvious. In the framework of the chosen test case and for equivalent error level, the non-conforming technique (4.3) uses about $\mathrm{e}^{0.5} \simeq 1.65$ times less nodes than the standard conforming method.

The last curve in Figure 3 represents the error measured when the penalty term has been dropped from (4.3). It appears that this method is no more converging which emphasizes the importance of the penalty term. When the penalty term is dropped, observe that the solution to (4.3) actually converges to a function satisfying the boundary condition (2.12) as well as the Helmoltz problem (2.11), but separately in $\Omega_{\mathrm{f}}$ and $\Omega_{\mathrm{c}}$ and with Neumann homogeneous conditions on both sides of the interface $\Gamma=\overline{\Omega_{\mathrm{f}}} \cap \overline{\Omega_{\mathrm{c}}}$. 
Acknowledgements. This work is supported by the Swiss National Science Foundation under the grant No. 2100-054156.98.

\section{REFERENCES}

[1] R.A. Adams, Sobolev Spaces. Academic Press, New-York, San Francisco, London (1975).

[2] F. Ben Belgacem, The mortar finite element method with Lagrange multipliers. Numer. Math. 84 (1999) 173-197.

[3] F. Ben Belgacem and Y. Maday, The mortar element method for three dimensional finite elements. RAIRO Modél. Math. Anal. Numér. 31 (1997) 289-302.

[4] M. Bercovier, Perturbation of mixed variational problems. Application to mixed finite element methods. RAIRO Anal. Numér. 12 (1978) 211-236.

[5] F. Brezzi and M. Fortin, Mixed and Hybride Finite Element Methods. Springer-Verlag, New York (1991).

[6] P.G. Ciarlet, The Finite Element Method for Elliptic Problem. North Holland, Amsterdam (1978).

[7] P. Clement, Approximation by finite element using local regularization. RAIRO Ser. Rouge 8 (1975) 77-84.

[8] P. Grisvard, Elliptic Problems in Nonsmooth Domains. Pitman, Boston (1985).

[9] J.L. Lions and E. Magenes, Problèmes aux limites non homogènes et applications, Vol. 1, Dunod, Paris (1968).

[10] Y. Maday, C. Bernardi and A.T. Patera, A new nonconforming approach to domain decomposition: the mortar element method, in Nonlinear Partial Differential Equations and their applications, H. Brezis and J.L. Lions Eds., Vol. XI, Pitman (1994) 13-51.

[11] J. Nitsche, Über eine Variationsprinzip zur Lösung von Dirichlet-Problemen bei Verwendung von Teilräumen, die keinen Randbedingungen unterworfen sind. Abh. Math. Sem. Univ. Hamburg 36 (1970/1971) 9-15.

[12] D. Schotzau, C. Schwab and R. Stenberg, Mixed hp-fem on anisotropic meshes ii. Hanging nodes and tensor products of boundary layer meshes. Numer. Math. 83 (1999) 667-697.

[13] R. Stenberg, On some techniques for approximating boundary conditions in the finite element method. J. Comput. Appl. Math. 63 (1995) 139-148.

To access this journal online: www.edpsciences.org 